\title{
Triangular and Hexagonal Tile Self-assembly Systems
}

\author{
Lila Kari, Shinnosuke Seki, and Zhi Xu \\ Department of Computer Science, University of Western Ontario, \\ London, Ontario, N6A 5B7 Canada
}

\begin{abstract}
We discuss theoretical aspects of the self-assembly of triangular tiles, in particular, right triangular tiles and equilateral triangular tiles, and the self-assembly of hexagonal tiles. We show that triangular tile assembly systems and square tile assembly systems cannot be simulated by each other in a non-trivial way. More precisely, there exists a deterministic square (hexagonal) tile assembly system $S$ such that no deterministic triangular tile assembly system that is a division of $S$ produces an equivalent supertile (of the same shape and same border glues). There also exists a deterministic triangular tile assembly system $T$ such that no deterministic square (hexagonal) tile assembly system produces the same final supertile while preserving border glues.
\end{abstract}

\section{Introduction}

A basic model of DNA computation by self-assembly was proposed by Adleman [1] and Winfree 2, based on the theory of Wang tiles [3. In this model, the basic components are square tiles with sides painted with "glues", that can stick together to form supertiles if the glues at abutting edges match, and attach with sufficient strength.

A regular tiling of the plane is a highly symmetric tiling made up of congruent regular polygons. Only three such regular tilings exist: those made up of equilateral triangles, squares, or hexagons. This paper departs from the existing model of self-assembly by investigating, instead of square tiles, the case of triangular tiles and hexagonal tiles. We namely discuss the self-assembly by equilateral-triangular, right-triangular, and hexagonal tile systems.

Our line of investigation follows that started by Winfree [4, who showed how the formation of large structures made out of the aggregation of rectangular DNA complexes can simulate Blocked Cellular Automata (BCA), which have the computational power of Turing machines. Winfree, Liu, Wenzler, and Seeman [2] designed and experimentally produced two-dimensional DNA crystals by self-assembly. A systematic study of self-assembly as a computational process was initiated by Adleman [1, who studied the time complexity of a particular case of linear self-assembly via "step counting" and raised the question of the construction of large squares via self-assembly. Rothemund and Winfree [5] studied the self-assembly of squares at fixed temperature (the threshold that the sum of the strengths of glues of a tile have to surpass, in order for it to "stick" to 


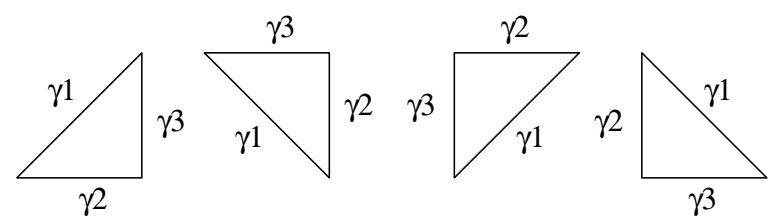

Fig. 1. Four kinds of isosceles right triangular tiles $\left(\gamma_{1}, \gamma_{2}, \gamma_{3}\right.$, se $),\left(\gamma_{1}, \gamma_{2}, \gamma_{3}\right.$, ne), $\left(\gamma_{1}, \gamma_{2}, \gamma_{3}, \mathrm{nw}\right)$, and $\left(\gamma_{1}, \gamma_{2}, \gamma_{3}, \mathrm{sw}\right)$

an existing assembled shape), and showed that in order to deterministically selfassemble an $N \times N$ full square (the square, $N$ tiles on a side), $N^{2}$ different tile types are required at temperature $\tau=1$ and $O(\log N)$ different tiles suffice at fixed temperature $\tau \geq 2$. Adleman, Cheng, Goel, and Huang [6] improved the latter result to $\Theta(\log N / \log \log N)$ different tiles. Kao and Schweller [7] showed that if the temperature $\tau$ is allowed to change systematically, then a constant number of tiles is enough for the self-assembly of an arbitrary $N \times N$ full square, with a temperature sequence of length $O(\log N)$.

In this paper, we follow a similar line of inquiry for triangular tiles and hexagonal tiles. Besides a natural theoretical interest, this study is motivated by the fact that triangular DNA tiles have been experimentally produced. For example, Liu, Wang, Deng, Walulu and Mao 8 reported the construction of a DNA triangle tile composed of three four-arm junctions, while Ding, Sha and Seeman 9 ] reported obtaining a triangular DNA tile formed from DX DNA molecules, and He, Chen, Liu, Ribbe and Mao [10] built a 3-point DNA star tile.

In this paper, in Sect. 2 we introduce the definition of triangular, respectively hexagonal, tile assembly systems. In Sect. 3 we compare the square tile assembly systems and triangular tile assembly systems from the point of view of shapes of the final supertiles they generate and show that the two types of systems cannot be simulated by each other in a straightforward way; we also compare the triangular tile assembly systems and hexagonal tile assembly systems.

\section{Preliminaries}

Our discussion of the triangular, respectively hexagonal, tile assembly systems will make use of the following definitions.

A triangular tile is a tile with three edges, each of which is "colored" with elements from a finite set $\Gamma$, called a glue set, whose elements dictate the interactions between the tiles. For all tiles discussed in this paper, we assume that the shortest side of the tile is of unit length, and that tiles cannot be rotated or flipped over.

An isosceles right triangular tile is a triangular tile in the shape of an isosceles right triangle, with each of its three edges colored by a glue from the glue set, and with the right angle pointing to the four possible directions: South-East, North-East, North-West, South-West as illustrated in Fig. 11. More formally, an 

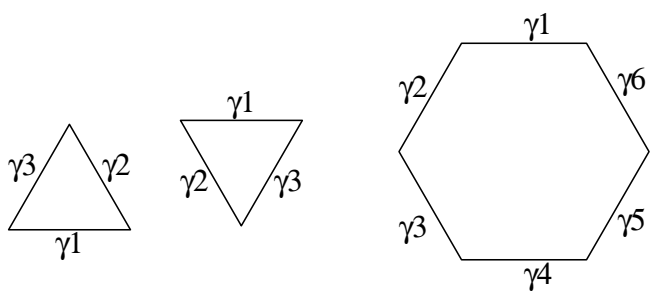

Fig. 2. Two equilateral triangular tiles $\left(\gamma_{1}, \gamma_{2}, \gamma_{3}, \mathrm{u}\right),\left(\gamma_{1}, \gamma_{2}, \gamma_{3}, \mathrm{~d}\right)$ and a hexagonal tile $\left(\gamma_{1}, \gamma_{2}, \gamma_{3}, \gamma_{4}, \gamma_{5}, \gamma_{6}\right)$
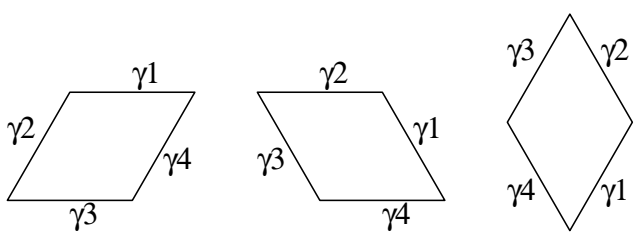

Fig. 3. Three diamond tiles $\left(\gamma_{1}, \gamma_{2}, \gamma_{3}, \gamma_{4}, \mathrm{II}\right),\left(\gamma_{1}, \gamma_{2}, \gamma_{3}, \gamma_{4}, \mathrm{IV}\right)$, and $\left(\gamma_{1}, \gamma_{2}, \gamma_{3}, \gamma_{4}, \mathrm{VI}\right)$

isosceles right triangular tile $t$ is represented as a quadruple $\left(\gamma_{1}, \gamma_{2}, \gamma_{3}, k\right)$, where $\gamma_{1}, \gamma_{2}, \gamma_{3} \in \Gamma$ are the glues on the sides of the tile in the counter-clockwise order starting from the longest side, and $k \in\{$ se, ne, nw, sw $\}$ presents the direction pointed to by the right angle. In the rest of this paper, we denote the glues $\gamma_{1}, \gamma_{2}, \gamma_{3}$ of the 3 edges of a tile $t$ by $\gamma_{1}(t), \gamma_{2}(t)$, and $\gamma_{3}(t)$. Throughout this paper we will call isosceles right triangular tiles simply right triangular tiles.

An equilateral triangular tile is a triangular tile in the shape of an equilateral triangle, with its edges colored by glues from the glue set, and that is either in an upward position or in a downward position as illustrated in Fig. 2, An equilateral triangular tile is formally represented as a quadruple $\left(\gamma_{1}, \gamma_{2}, \gamma_{3}, k\right)$, where $\gamma_{1}, \gamma_{2}, \gamma_{3} \in \Gamma$ are the glues on the sides of the tile in the counter-clockwise order starting from the horizontal side and $k \in\{\mathrm{u}, \mathrm{d}\}$ presents the upward, respectively downward, orientation of the "arrow" represented by the triangle. The notations $\gamma_{1}(t), \gamma_{2}(t)$, and $\gamma_{3}(t)$ are defined in the same way as for right triangular tiles.

A regular hexagonal tile is a tile in the shape of a regular hexagon, with each of the six edges being colored with glues from the set $\Gamma$. Unlike triangular tiles, two geometrically adjacent regular hexagonal tiles must be of the same orientation. Without loss of generality, we assume that all regular hexagonal tiles are positioned as illustrated in Fig. 2, More formally, a regular hexagonal tile $t$ is represented as a tuple $\left(\gamma_{1}, \gamma_{2}, \gamma_{3}, \gamma_{4}, \gamma_{5}, \gamma_{6}\right)$, where $\gamma_{i} \in \Gamma$ are the glues on the sides of the tile in the counter-clockwise order starting from the top-most side. The notations $\gamma_{i}(t)$ for $i=1, \ldots, 6$ are defined in the same way as for triangular tiles. In this paper we will only investigate regular hexagonal tiles, and simply call them hexagonal tiles. 


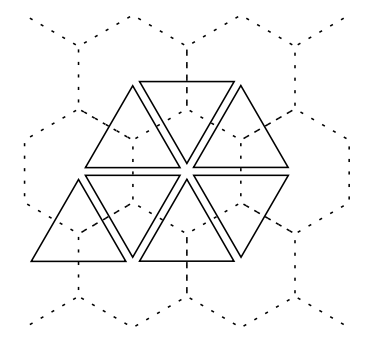

Fig. 4. A hexagonal grid graph

A diamond tile is a tile in the shape of a diamond (rhombus), one angle of which is $\pi / 3$, with each of the four edges being colored with glues from the set $\Gamma$. We assume each diamond tile is in one of the three possible positions illustrated in Fig. 3. More formally, a diamond tile $t$ is represented as a tuple $\left(\gamma_{1}, \gamma_{2}, \gamma_{3}, \gamma_{4}, k\right)$, where $\gamma_{i} \in \Gamma$ are the glues on the sides of the tile and $k \in$ $\{$ II, IV, VI $\}$ as specified by the three typical examples in Fig. 3, The notations $\gamma_{i}(t)$ for $i=1, \ldots, 4$ are defined in the same way as for triangular tiles.

Let us define $\mathcal{T}_{\mathrm{sq}}=\Gamma^{4}, \mathcal{T}_{\mathrm{R}}=\Gamma^{3} \times\{\mathrm{se}, \mathrm{ne}, \mathrm{nw}, \mathrm{sw}\}, \mathcal{T}_{\triangle}=\Gamma^{3} \times\{\mathrm{u}, \mathrm{d}\}, \mathcal{T}_{\mathrm{H}}=$ $\Gamma^{6}$, and $\mathcal{T}_{\mathrm{D}}=\Gamma^{4} \times\{I I, I V, V I\}$ as the sets of all possible square tiles, right triangular tiles, equilateral triangular tiles, hexagonal tiles, and diamond tiles respectively, given the glue set $\Gamma$. We can further split $\mathcal{T}_{\mathrm{R}}$ into four disjoint subsets $\mathcal{T}_{\mathrm{R}, \mathrm{se}}, \mathcal{T}_{\mathrm{R}, \mathrm{ne}}, \mathcal{T}_{\mathrm{R}, \mathrm{nw}}, \mathcal{T}_{\mathrm{R}, \mathrm{sw}}$ depending on the fourth element of tiles, defined as: $\mathcal{T}_{\mathrm{R}, x}=\left\{\left(\gamma_{1}, \gamma_{2}, \gamma_{3}, x\right) \in \mathcal{T}_{\mathrm{R}}\right\}$ for $x \in\{\mathrm{se}, \mathrm{ne}, \mathrm{nw}, \mathrm{sw}\}$. In a similar manner, $\mathcal{T}_{\triangle}$ can be split into the two disjoint subsets $\mathcal{T}_{\triangle, \mathrm{u}}, \mathcal{T}_{\triangle, \mathrm{d}}$ and $\mathcal{T}_{\mathrm{D}}$ can be split into three disjoint subsets $\mathcal{T}_{\mathrm{D}, \mathrm{II}}, \mathcal{T}_{\mathrm{D}, \mathrm{IV}}, \mathcal{T}_{\mathrm{D}, \mathrm{vI}}$.

Let us proceed now to augment the notion of glue by associating to every glue a numerical "glue strength" as follows. Let $\mathcal{R}$ be the set of non-negative real numbers. Let $\Gamma=\left\{\left(\ell_{1}, n_{1}\right),\left(\ell_{2}, n_{2}\right), \ldots,\left(\ell_{k}, n_{k}\right) \mid n_{1}, \ldots, n_{k} \in \mathcal{R}\right.$, where $\ell_{1}, \ldots, \ell_{k}$ are unique labels, i.e., $\ell_{i}=\ell_{j}$ iff $\left.i=j\right\}$ for some $k \geq 1$. The set $\Gamma$ dictates interactions between tiles, where for each $1 \leq i \leq k, \ell_{i}$ is the label of the $i$-th glue and $n_{i}$ is the glue strength associated with it. (In the figures of this paper, the strength associated with the glue on a side will be represented by the number of parallel edges along that side.) A particular glue $\phi \in \Gamma$, defined as $\phi=(\phi, 0)$, denotes the non-interactive glue. Tiles can stick to each other by the glues on their adjacent edges to form supertiles.

Let $T$ be a set of tiles of the same kind (square, equilateral triangle, right triangle, hexagon, or diamond). Conventionally, tiling the plane by tiles in $T$ is modeled as a partial function from either the set of coordinates on the plane, or from the corresponding undirected lattice graph, to the set of tiles $T$. This partial function is called a supertile of $T$. Tiles assigned to adjacent vertices of the lattice graph are considered to be adjacent in the supertile.

For square supertiles, the coordinate system should be orthogonal, and hence, the corresponding graph is the grid graph (the two-dimensional integer lattice). In contrast, the lattice graph for a supertile made of triangular tiles should 


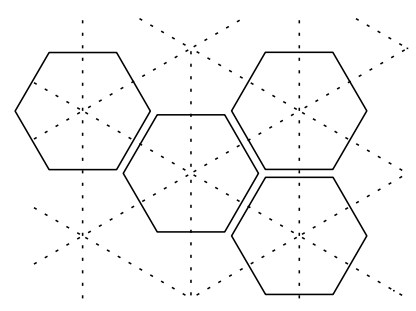

Fig. 5. A triangular grid graph

be 3-regular (each vertex of the underlying graph has 3 neighbours) because a triangular tile can abut to at most 3 other tiles. Thus, the most appropriate lattice graph for tiling by equilateral triangular tiles is a hexagonal grid graph $H=(V, E)$ (see Fig. (4). In order to enforce the condition that two upward equilateral triangular tiles are never adjacent to each other, and neither are two downward ones, $H$ has to be bipartite as: $V=V_{\mathrm{u}} \cup V_{\mathrm{d}}$ and $E \subseteq V_{\mathrm{u}} \times V_{\mathrm{d}}$. For $T \subseteq \mathcal{T}_{\triangle}$, a supertile $C$ of $T$ is defined as a partial function from $V$ to $T$ such that

1. for any $t_{\mathrm{u}} \in \mathcal{T}_{\triangle, \mathrm{u}}$, if $C(v)=t_{\mathrm{u}}$, then $v \in V_{\mathrm{u}}$, and

2. for any $t_{\mathrm{d}} \in \mathcal{T}_{\triangle, \mathrm{d}}$, if $C\left(v^{\prime}\right)=t_{\mathrm{d}}$, then $v^{\prime} \in V_{\mathrm{d}}$.

For defining a supertile of a set of right triangular tiles, the underlying 3-regular lattice graph $G=\left(V^{\prime}, E^{\prime}\right)$ should be a 4-partite graph because there are the four kinds of right triangular tiles. Hence, let $V^{\prime}=V_{\mathrm{se}} \cup V_{\mathrm{ne}} \cup V_{\mathrm{nw}} \cup V_{\mathrm{sw}}$ and $E^{\prime}$ satisfy

1. for $v_{\text {se }} \in V_{\text {se }},\left\{\left(v_{\text {se }}, v_{1}\right),\left(v_{\text {se }}, v_{2}\right),\left(v_{\text {se }}, v_{3}\right)\right\} \subseteq E^{\prime}$ such that $v_{1} \in V_{\text {nw }}, v_{2} \in$ $V_{\mathrm{ne}} \cup V_{\mathrm{nw}}$, and $v_{3} \in V_{\mathrm{nw}} \cup V_{\mathrm{sw}}$;

2. for $v_{\text {ne }} \in V_{\text {ne }},\left\{\left(v_{\text {ne }}, v_{1}^{\prime}\right),\left(v_{\text {ne }}, v_{2}^{\prime}\right),\left(v_{\text {ne }}, v_{3}^{\prime}\right)\right\} \subseteq E^{\prime}$ such that $v_{1}^{\prime} \in V_{\text {sw }}, v_{2}^{\prime} \in$ $V_{\mathrm{nw}} \cup V_{\mathrm{sw}}$, and $v_{3}^{\prime} \in V_{\mathrm{se}} \cup V_{\mathrm{sw}}$;

3. for $v_{\mathrm{nw}} \in V_{\mathrm{nw}},\left\{\left(v_{\mathrm{nw}}, v_{1}^{\prime \prime}\right),\left(v_{\mathrm{nw}}, v_{2}^{\prime \prime}\right),\left(v_{\mathrm{nw}}, v_{3}^{\prime \prime}\right)\right\} \subseteq E^{\prime}$ such that $v_{1}^{\prime \prime} \in V_{\mathrm{se}}, v_{2}^{\prime \prime} \in$ $V_{\mathrm{se}} \cup V_{\mathrm{sw}}$, and $v_{3}^{\prime \prime} \in V_{\mathrm{se}} \cup V_{\mathrm{ne}}$;

4. for $v_{\mathrm{sw}} \in V_{\mathrm{sw}},\left\{\left(v_{\mathrm{sw}}, v_{1}^{\prime \prime \prime}\right),\left(v_{\mathrm{sw}}, v_{2}^{\prime \prime \prime}\right),\left(v_{\mathrm{sw}}, v_{3}^{\prime \prime \prime}\right)\right\} \subseteq E^{\prime}$ such that $v_{1}^{\prime \prime \prime} \in V_{\mathrm{ne}}, v_{2}^{\prime \prime \prime} \in$ $V_{\mathrm{se}} \cup V_{\mathrm{ne}}$, and $v_{3}^{\prime \prime \prime} \in V_{\mathrm{ne}} \cup V_{\mathrm{nw}}$.

For $T^{\prime} \subseteq \mathcal{T}_{\mathrm{R}}$, a supertile $C^{\prime}$ of $T^{\prime}$ is defined as a partial function from $V^{\prime}$ of $G$ to $T^{\prime}$ such that for any $t_{x} \in \mathcal{T}_{\mathrm{R}, x}$, if $C^{\prime}(v)=t_{x}$, then $v \in V_{x}$, where $x \in\{$ se, ne, nw, sw $\}$. For defining the supertile of a set of hexagonal tiles, a 6-regular lattice graph, triangular grid graph $\operatorname{Tr}=\left(V^{\prime \prime}, E^{\prime \prime}\right)$, is adopted (see Fig. (5). For $T^{\prime \prime} \subseteq \mathcal{T}_{\mathrm{H}}$, a supertile $C^{\prime \prime}$ of $T^{\prime \prime}$ is defined as a partial function from $V^{\prime \prime}$ of $\operatorname{Tr}$ to $T^{\prime \prime}$. For defining the supertile of a set of diamond tiles, a 4-regular lattice graph $G^{\prime}=\left(V^{\prime \prime \prime}, E^{\prime \prime \prime}\right)$ is needed and the lattice graph is a 3-partite graph. Let $V^{\prime \prime \prime}=V_{\mathrm{II}} \cup V_{\mathrm{IV}} \cup V_{\mathrm{VI}}$ and $E^{\prime \prime \prime}$ satisfy. 
1. for $v_{\mathrm{II}} \in V_{\mathrm{II}},\left\{\left(v_{\mathrm{II}}, v_{1}\right),\left(v_{\mathrm{II}}, v_{2}\right),\left(v_{\mathrm{II}}, v_{3}\right),\left(v_{\mathrm{II}}, v_{4}\right)\right\} \subseteq E^{\prime \prime \prime}$ such that $v_{1}, v_{3} \in$ $V_{\mathrm{II}} \cup V_{\mathrm{IV}}$ and $v_{2}, v_{4} \in V_{\mathrm{II}} \cup V_{\mathrm{VI}}$;

2. for $v_{\mathrm{IV}} \in V_{\mathrm{IV}},\left\{\left(v_{\mathrm{IV}}, v_{1}\right),\left(v_{\mathrm{IV}}, v_{2}\right),\left(v_{\mathrm{IV}}, v_{3}\right),\left(v_{\mathrm{IV}}, v_{4}\right)\right\} \subseteq E^{\prime \prime \prime}$ such that $v_{2}, v_{4} \in$ $V_{\text {II }} \cup V_{\text {IV }}$ and $v_{1}, v_{3} \in V_{\text {IV }} \cup V_{\mathrm{VI}}$;

3. for $v_{\mathrm{VI}} \in V_{\mathrm{VI}},\left\{\left(v_{\mathrm{VI}}, v_{1}\right),\left(v_{\mathrm{VI}}, v_{2}\right),\left(v_{\mathrm{VI}}, v_{3}\right),\left(v_{\mathrm{VI}}, v_{4}\right)\right\} \subseteq E^{\prime \prime \prime}$ such that $v_{1}, v_{3} \in$ $V_{\mathrm{II}} \cup V_{\mathrm{II}} \cup V_{\mathrm{VI}}$ and $v_{2}, v_{4} \in V_{\mathrm{IV}} \cup V_{\mathrm{VI}}$.

For $T^{\prime \prime \prime} \subseteq \mathcal{T}_{\mathrm{D}}$, a supertile $C^{\prime \prime \prime}$ of right triangular tiles from $T^{\prime \prime \prime}$ is defined as a partial function from $V^{\prime \prime \prime}$ of $G^{\prime}$ to $T^{\prime \prime \prime}$. In the definition of a supertile, both the hexagonal grid graph $H$ and the triangular grid graph $\mathrm{Tr}$ are unique, but there are more than one valid lattice graphs to present supertiles for right triangular tiles and for diamond tiles.

Let us now formally define the interaction between tiles which depends on the match between the glues on the tiles' adjacent edges and also on a threshold parameter called temperature $\tau \in \mathcal{R}$ that determines whether or not the "sticking" is strong enough for the new tile to attach to an existing supertile. In general, the strength function $g: \Gamma \times \Gamma \rightarrow \mathcal{R}$ is defined such that $g\left(\gamma, \gamma^{\prime}\right)=g\left(\gamma^{\prime}, \gamma\right)$ and $g(\phi, \gamma)=0$ for all $\gamma, \gamma^{\prime} \in \Gamma$. In particular, we are interested in the discrete case where $\tau$ is an integer and $g\left((\ell, n),\left(\ell^{\prime}, n^{\prime}\right)\right)=n$ if $\ell=\ell^{\prime}$ and $n=n^{\prime} ; g\left((\ell, n),\left(\ell^{\prime}, n^{\prime}\right)\right)=0$ otherwise. We call a supertile $D$ full if the strength $g\left(\gamma_{i}(D(v)), \gamma_{i}\left(D\left(v_{i}\right)\right)\right)$ of common edges of every two adjacent tiles $D(v)$ and $D\left(v_{i}\right)$ in the supertile is strictly positive.

In order to model the growth of tile assemblies, we need to define the notion of attachability. Let $T \subseteq \mathcal{T}_{\triangle}$ be a set of equilateral triangular tiles and $C, D$ be two supertiles of $T$. We say that $t$ attaches to $C$ at vertex $v$, to derive $D$, and we write $C \rightarrow_{T, g, \tau} D$, if the following conditions hold. Firstly, $C(u)=D(u)$ for all $u \in$ $\operatorname{dom}(C)$. Secondly, there exist some $t \in T$ and $v \in V$ such that $C(v)$ is undefined, $\operatorname{dom}(C)=\operatorname{dom}(D) \backslash\{v\}, D(v)=t$, and for every $\left\{\left(v, v_{1}\right),\left(v, v_{2}\right),\left(v, v_{3}\right)\right\} \subseteq E$ we have,

$$
\sum_{i \in\{1,2,3\}} g\left(\gamma_{i}(t), \gamma_{i}\left(D\left(v_{i}\right)\right)\right) \geq \tau .
$$

Informally, the supertile $D$ is derived from the supertile $C$ by the attachment of $t$ to $C$ iff the sum of the glue strengths on those edges of $t$ that are adjacent to $C$ is greater than or equal to the threshold $\tau$. Note that in the definition of attachability, we do not require either $C$ or $D$ be full.

We can define the notions of attachability and transition for right triangular tiles, hexagonal tiles and diamond tiles in a similar manner, and those notions for square tiles can be found in the literature [6]. The reflexive and transitive closure of $\rightarrow_{T, g, \tau}$ is denoted by $\rightarrow_{T, g, \tau}^{*}$.

A tile assembly system (TAS) is a tuple $S=(T, s, g, \tau)$. $T$ is a finite set of tiles of the same kind; so $T \subseteq \mathcal{T}_{\text {sq }}\left(T \subseteq \mathcal{T}_{\mathrm{R}}, T \subseteq \mathcal{T}_{\triangle}, T \subseteq \mathcal{T}_{\mathrm{H}}, T \subseteq \mathcal{T}_{\mathrm{D}}\right)$ implies that all tiles of $S$ are square (respectively, right triangular, equilateral triangular, hexagonal, diamond). The other parameters of $S$ mean that $s \in T$ is a special supertile called the seed, $g$ is a strength function, and $\tau$ is the temperature.

We now define the notion of derived supertile of a given TAS $S$ as follows. The seed tile $s$, when placed in an a priori chosen "reference position" on the 


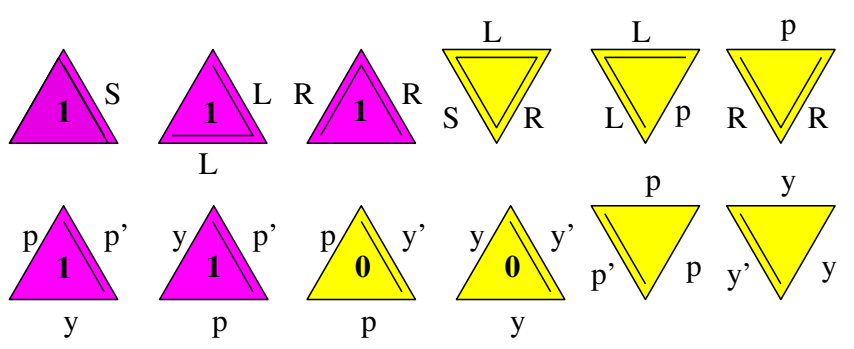

Fig. 6. A set of 12 tiles from which the Sierpinski triangle self-assembles deterministically. $S, L, R, p, p^{\prime}, y$, and $y^{\prime}$ are the glue labels and the number of parallel lines along each edge denotes the glue strength. The labels 1 and 0 specify the digits used in the XOR operation.

plane or on the grid graph, is a partial function called the seed derived supertile or simply seed supertile. For example, in the case of square TASs, we may choose to always place the seed supertile on the plane as the square with corners at coordinates $(0,0),(0,1),(1,0),(1,1)$. A derived supertile of $S$ is a supertile $C$ such that $s \rightarrow_{T, g, \tau}^{*} C$. A final supertile of $S$ is a derived supertile $C$ such that $C \rightarrow_{T, g, \tau}^{*} D$ implies $C=D$ for any supertile $D$, that is, no tile is attachable at any vertex in $C$. The number of tile types of $S$ is called the program size complexity of $S$, and is denoted by $|S|[5$.

A TAS is said to be deterministic if its final supertile is unique regardless of how the self-assembly proceeds starting from the seed. Otherwise, the TAS is said to be non-deterministic. A non-deterministic TAS can have many different final supertiles possibly with different shapes. In this paper, unless explicitely stated otherwise, all tile systems are assumed to be deterministic. When $T \subseteq \mathcal{T}_{\mathrm{R}}$ (respectively, $T \subseteq \mathcal{T}_{\triangle}, T \subseteq \mathcal{T}_{\mathrm{H}}, T \subseteq \mathcal{T}_{\mathrm{D}}$ ), $S$ is explicitly called a right triangular $T A S$ (respectively, an equilateral triangular TAS, a hexagonal TAS, a diamond TAS).

Before we discuss shapes generated by non-square self-assembly systems, let us first see an example of how a Sierpinski triangle can self-assemble using tiles from an equilateral triangular TAS.

Proposition 1. There exists an equilateral triangular tile system which deterministically self-assembles the Sierpinski triangle at temperature $\tau=2$.

Proof. Let us recall the square tile system which deterministically self-assembles the Sierpinski triangle [11, whose tile set contains seven tile types in two colour categories, dark and light. Using the technique of "division", formally defined in Section 3 we transform each of the square tiles by flattening it into a parallelogram, and dividing this parallelogram to obtain one upward and one downward equilateral triangular tile, see Fig. 9. Furthermore, by reusing some of the triangular tiles, the number of triangular tiles needed to assemble an arbitrarily large Sierpinski triangle is reduced to twelve. The tile set of an equilateral triangular tile system thus designed is illustrated in Fig. 6. 
The seed tile is $(\phi, S, \phi, \mathrm{u})$ and together with the other five tiles on the top row in Fig. 6 can form the $L$-shaped supertile, which composes the outmost left and bottom boundary of the Sierpinski triangle (See Fig. 7). Then each parallelogram-shaped space is filled by a pair of triangular tiles that together simulate a flattened square tile that implements an XOR-like rule. This XOR rule takes as input the left and bottom glues of the first triangular tile of the pair (corresponding to the left and bottom neighbours of that tile), and outputs the result as the right and top glues of the second triangular tile of the pair. The tiles of the pair are held together by a glue of strength 2 . Four tile types are enough to implement the XOR operation. Two more tile types deliver the information and fill the triangle, as illustrated on the bottom row in Fig. 6. The result is a Sierpinski triangle as illustrated in Fig. 7.

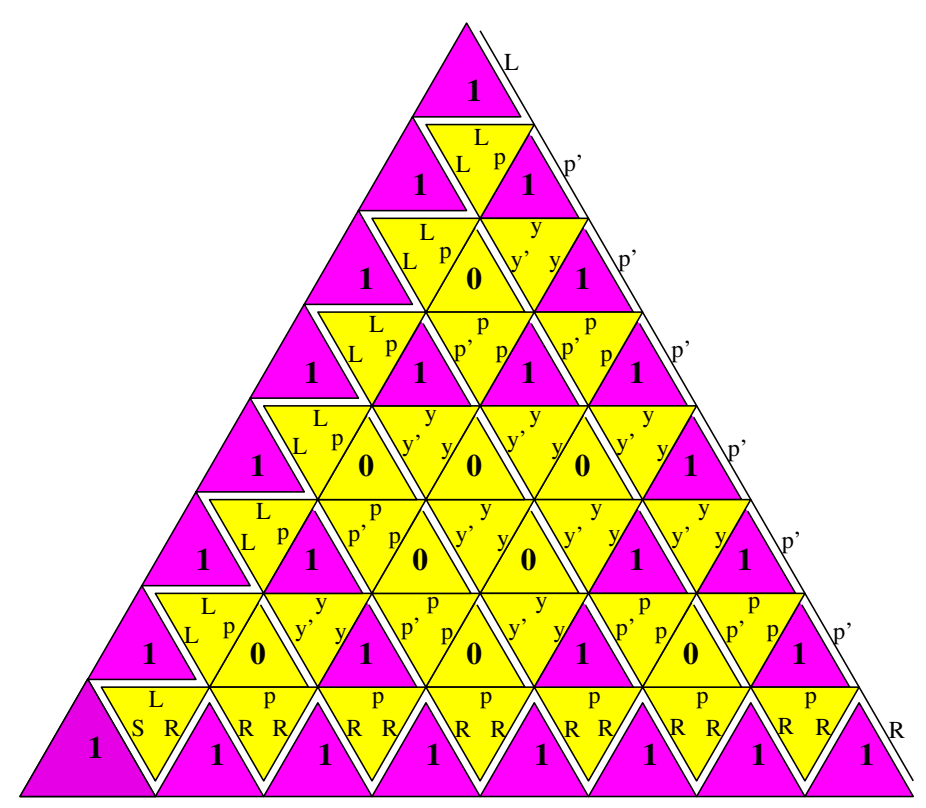

Fig. 7. The Sierpinski-triangle which consists of 64 equilateral triangular tiles with 12 different tile types

\section{Comparing Supertiles}

We now proceed to compare the final supertiles produced by various tile assembly systems in terms of their shape and boundary glues.

The tiles we considered are well defined geometrically by their shape and the fact that their shortest edge is of unit length. Thus, given a TAS with tiles of shape $\alpha$, where $\alpha \in\{$ square, triangle, right triangle, hexagon\}, we can now associate to every supertile a corresponding region in $\mathcal{R}^{2}$ as follows. 
We associate to a supertile of size 1, i.e., consisting of one tile only, the region in $\mathcal{R}^{2}$ enclosed by the edges of that tile, assuming the tile is placed on the twodimensional plane at an a priori chosen reference position. For example if $\alpha=$ square, then the corresponding region is the square (including its interior) with corners $(0,0),(0,1),(1,0),(1,1)$. Let us assume we have associated to the seed tile a region in $\mathcal{R}^{2}$ in this fashion. We can now associate to a supertile of size 2 , obtained by attaching a single tile to the seed tile, a region in $\mathcal{R}^{2}$. This is obtained by taking the union between the region in $\mathcal{R}^{2}$ coresponding to the seed tile, and the region in $\mathcal{R}^{2}$ resulting by translating the region in $\mathcal{R}^{2}$ corresponding to the second tile to the position where it attaches to the seed. By iterating the process, we can thus associate to each supertile that is derived from the seed a corresponding region in $\mathcal{R}^{2}$.

Two supertiles are said to have the same shape if their corresponding regions of $\mathcal{R}^{2}$ are identical. If, in addition, the glues on the boundaries of the two supertiles (but not necessarily the internal glues) are the same, the two supertiles are said to be equivalent. The fact that, in order to be considered equivalent, final supertiles have the same boundary glues in addition to covering the same region in $\mathcal{R}^{2}$, reflects the fact that supertiles are often used as components for further assemblies, and thus have to have the same "sticking properties" if they are to be used interchangeably.

A region $Y \subseteq \mathcal{R}^{2}$ is called $\alpha$-compatible, where $\alpha$ is an element of s square, right triangle, equilateral triangle, hexagon, diamond $\}$, if $Y$ can be geometrically "covered" by tiles from an $\alpha$-TAS, i.e., if $Y$ can be written as the set union of regions, overlapping at most on their edges, that are obtained by translating the regions corresponding to single tiles from an $\alpha$-TAS. We call a supertile $\alpha$-compatible if its corresponding region in $\mathcal{R}^{2}$ is $\alpha$-compatible.

For example, the region corresponding to the final supertile of any hexagonal TAS is equilateral-triangle-compatible, and none of the triangular regions of $\mathcal{R}^{2}$ is square-compatible. For a given $\alpha$-TAS, only the assembly of final supertiles of $\alpha$-compatible shapes is meaningful. Hence, in the remaining discussion, we only consider the assembly of final supertiles of $\alpha$-compatible shapes.

First, note that for any $\alpha$-compatible supertile, there is a trivial $\alpha$-TAS (deterministic or non-deterministic) that produces a final supertile of the same shape.

Proposition 2. Let $\alpha \in\{$ square, right triangle, equilateral triangle, hexagon, diamond $\}$ and let $Y$ be an $\alpha$-compatible supertile. There exists a nondeterministic $\alpha$-TAS of a constant number of tile types whose final supertile has the same shape as $Y$. If $Y$ is finite, then there exists a deterministic $\alpha-T A S$ with $n$ tile types whose final supertile has the same shape as $Y$, where $n$ is the total number of tiles needed to mosaic $Y$ geometrically.

Proof. Let us consider $\alpha$ being equilateral triangle. The other cases are similar. Consider first the non-deterministic case. Let $T$ be the set of tiles $T=$ $\{(a, b, c, k) \mid a, b, c \in\{\phi, g\}, k \in\{\mathrm{u}, \mathrm{d}\}\}$. All glues of $g$ are of strength 1 and temperature is $\tau=1$. The seed and assembly process are as follows: The supertile $Y$ is assembled according to the geometrical division of the region enclosed 


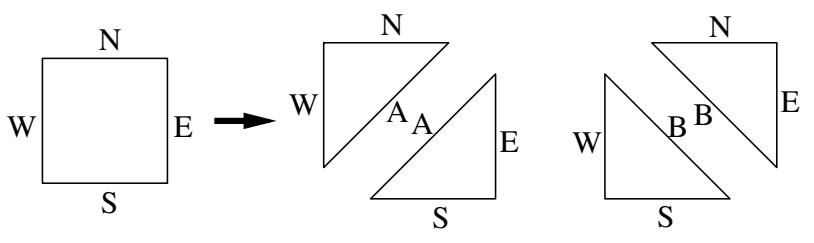

Fig. 8. Two ways to divide a square tile into right triangular tiles

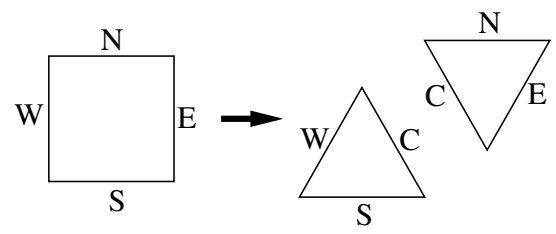

Fig. 9. Even with the help of affine transformations, squares can be divided into two equilateral triangles in only one way

by $Y$ into triangular tiles of $T$. This can be done since $Y$ is equilateral-trianglecompatible. At each step, a tile sticks to the supertile in such a way that if the tile is surrounded by other tiles in the completed region, then every edge of that tile is of glue $g$; otherwise, the edge that compose the boundary of that region are of empty glue $\phi$. Then a final supertile of the same shape as $Y$ can be produced by the given TAS with at most 16 tile types.

For the deterministic case, we mosaic $T$ geometrically with equilateral triangular tiles. If $n$ tiles are needed, we define a TAS consisting of $n$ tiles, where the glues between each two tiles that stick to each other in the final supertile are unique.

Proposition 2 shows that, if we are interested only in shape, and we either a) do not care that a unique supertile is assembled, or $b$ ) we care about uniqueness of the final supertile but we do not care about the program complexity of the tile system (how many tile types are needed), then $\alpha$-compatible tile systems can essentially produce final supertiles of the same shape. This is because every $\alpha$-compatible supertile can be produced by a trivial but huge deterministic $\alpha$ TAS of type $\alpha$, where $\alpha \in$ \{square, right triangle, equilateral triangle, hexagon, diamond $\}$, or by a non-deterministic one. In what follows, we will discuss natural restrictions on the comparison of TASs, that avoid these trivial cases.

We first compare the triangular TASs and square TASs from the point of view of the shapes of the final supertiles they generate. A right triangular TAS $S_{\mathrm{R}}=\left(T_{\mathrm{R}}, s_{\mathrm{R}}, g, \tau\right)$ is called a triangular division of a square TAS $S=(T, s, g, \tau)$, if:

1. For any square tile $t \in T$ of $S$, there is a pair of triangular tiles $t^{\prime}, t^{\prime \prime} \in T_{\mathrm{R}}$ of $S_{\mathrm{R}}$ whose hypotenuses are colored with the same glue $\left(l_{t}, n_{t}\right)$ with $n_{t} \geq \tau$ 


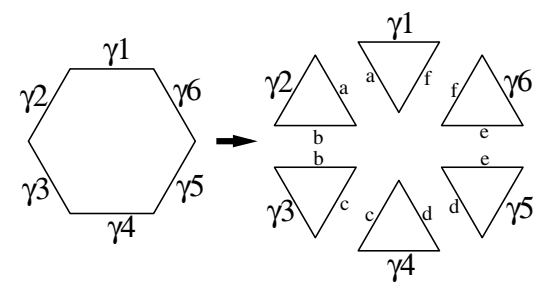

Fig. 10. A hexagonal tile is divided into six equilateral triangular tiles

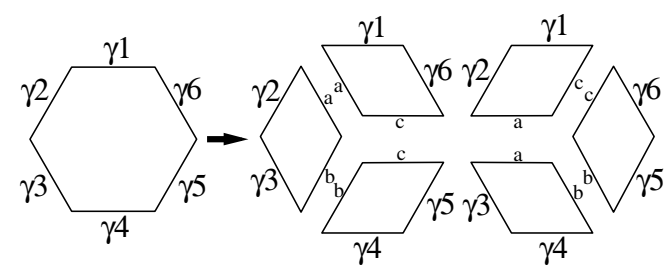

Fig. 11. A hexagonal tile is divided into three diamond tiles in two different ways

so that at temperature $\tau \geq 1$, these tiles can stick to each other via their hypotenuses and result in a two-tile supertile equivalent to the square tile $t$;

2. For any triangular tile $t \in T_{\mathrm{R}}$, there exists another triangular tile $t^{\prime} \in T_{\mathrm{R}}$ of $S_{\mathrm{R}}$ such that these two tiles can stick to each other via their hypotenuses and produce a supertile that is equivalent to a square tile in $T$ at temperature $\tau \geq 1$.

Note that the "hypothenuse glues" may or may not be distinct for different triangular tile pairs. Note also that the numbers of tiles in the two systems, the square tile system $S$ and its division $S_{R}$, above satisfy the inequality $\sqrt{|T|} \leq$ $\left|T_{R}\right|$. By definition, the division of a square TAS may not be unique. This is mainly because a square tile can be divided into two right triangular tiles in two different ways (see Fig. 8). In addition, two different square TASs can have the same right triangular TAS as a division. Finally, note that even if a square TAS is deterministic, its triangular division may not be so. A triangular division of a square TAS $S$ is called a deterministic triangular division if it is a triangular division of $S$ and, in addition, it is deterministic.

Let us define the flattening function $f: \mathcal{T}_{\triangle} \rightarrow \mathcal{T}_{\mathrm{R}}$ as $f\left(\left(\gamma_{1}, \gamma_{2}, \gamma_{3}, \mathrm{u}\right)\right)=$ $\left(\gamma_{2}, \gamma_{3}, \gamma_{1}\right.$, sw $)$ and $f\left(\left(\gamma_{1}, \gamma_{2}, \gamma_{3}, \mathrm{~d}\right)\right)=\left(\gamma_{2}, \gamma_{3}, \gamma_{1}\right.$, ne $)$. This function has the effect of "flattening" an equilateral triangular TAS $S=(T, s, g, \tau)$ into a right triangular TAS $\mathcal{F}(S)=(U, f(s), g, \tau)$, where $U=\{f(t) \mid t \in T\}$. Informally, a flattened right-triangular TAS is obtained from an equilateral triangular one by morphing each of the equilateral triangular tiles into either a South-West pointing, or respectively North-East pointing right triangular one.

An equilateral triangular TAS $T$ is called a division of a square $T A S S$, Fig. 9, if the flattened TAS $\mathcal{F}(T)$ obtained from it is a division of $S . T$ is called a 
deterministic division of $S$ if it is a division of $S$ which is deterministic. The numbers of tiles in the two systems, the square tile system $S$ with a tile set $T_{S}$ and its equilateral triangle division $T$ with the tile set $T_{T}$, satisfy the inequality $\sqrt{\left|T_{S}\right|} \leq\left|T_{T}\right|$.

We now ask the question of whether or not any square TAS can be converted, by division, into a triangular TAS that produces an equivalent final supertile. In general, the answer is "no", as shown by the following lemma.

Lemma 1. There exists a deterministic square TAS, none of whose deterministic triangular divisions produces an equivalent final supertile.

Two examples proving this lemma are illustrated in Fig. 13, one for $\tau=2$, (left), and one for $\tau=3$, (center). In the figure, each tile is numbered in the order of a possible assembly process.

For the example in Fig. 13 (left), each of the square tiles $s, 1, \ldots, 6$ can be simulated by a pair of right triangular tiles. There are two sticky edges for tile 7 , which are on parallel sides of the square tile, each of which is of strength 1 . So under $\tau=2$ the attachment of tile 7 cannot be simulated by successive attachments of two right triangular tiles to assemble the same final supertile. This is because the edges necessary for tile 7 to attach are its North and South edges, both of them of strength one. No matter how we divide this square tile into two triangles, the North and South edges will belong to different triangular tiles and, because they have only strength $1 \leq \tau=2$, neither of them can attach to the existing supertile.

The next example is the $4 \times 4$ square in Fig. 13 (center). By a similar reasoning, the attachment of tile 11 cannot be simulated by successive attachments of two right triangular tiles, and thus, the assembly stops and fails to grow into the $4 \times 4$ square.

The supertile in Fig. 13 (left) has a missing tile in the middle, and we say that it has a "hole". In general, a derived supertile $S$ is hole-free if it is full and for any closed tile-path, all the positions of the grid subgraph corresponding to $S$ that are inside the closed path are filled with tiles.

Lemma 2. For any deterministic square TAS $S$ at $\tau=1$, and any square TAS at $\tau=2$ whose final supertile is hole-free, there is a triangular division of $S$ that can produce an equivalent final supertile.

Proof. For $\tau=1$, the proof is straightforward. Any square tile $s_{i}$ with glues $\gamma_{1}, \gamma_{2}, \gamma_{3}, \gamma_{4}$ (on East, North, West, South sides, respectively) in $S$ is replaced with a pair of right triangular tiles $\left(i, \gamma_{1}, \gamma_{2}\right.$, ne) and $\left(i, \gamma_{3}, \gamma_{4}, \mathrm{sw}\right)$, where $i$ is a new glue added, and when $s_{i}$ is the seed, we let $\left(i, \gamma_{1}, \gamma_{2}\right.$, ne) be the new seed. Then the new right triangular TAS is a division of $S$ and produces an equivalent final supertile.

Now we assume $\tau=2$ and assume that there is no hole in the final supertile of $S$.

First we prove that for any hole-free derived supertile st, there is an assembly process such that every derived supertile in the process is hole-free. For an 


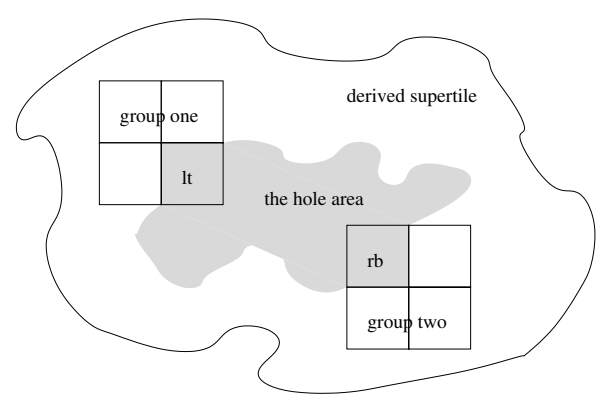

Fig. 12. Proof of Lemma 22 Tiles $l t$ and $r b$ in a hole area

assembly process $p: s t_{0} \rightarrow s t_{1} \rightarrow s t_{2} \rightarrow \ldots \rightarrow s t_{n}=s t$, let $f(p)$ be the number such that $s t_{i}$ is hole-free for $i<f(p)$ but $s t_{f(p)}$ has a hole. For the case that none of $s i_{i}$ has a hole, we write $f(p)=+\infty$. Now choose a $p$ such that $f(p)$ is the largest among all assembly process of st. We prove by contradiction and assume $f(p) \neq+\infty$. Let $t$ be the new attached tile in the step $s t_{f(p)-1} \rightarrow s t_{f(p)}$. Since $s t_{n}$ is full, every $s t_{i}$ is also full. So $s t_{f(p)}$ has a hole for the reason that there are missing tiles in a hole region. Those missing tiles will eventually be filled up in $s t_{n}$ since $s t_{n}$ is hole-free. Now we consider the following two tiles, not necessarily distinct, among all missing tiles in the hole of $s t_{f(p)}$ : the left-most tiles $l t$ among the top-most tiles, and the right-most tiles $r b$ among the bottommost tiles. Then the closest positions to the North of $l t$, to the West of $l t$ and to the North-West of $l t$ have tiles on them, called group one, and similarly the closest positions to the South of $r b$, to the East of $r b$ and to the South-East of $r b$ have tiles on them, called group two. (See Fig. 12) Tile $t$ cannot be in both group one and group two. Without loss of generality, we assume $t$ is not in group one. So both West and North of $l t$ are tiles in $s t_{f(p)-1}$, and thus there are two adjacent edges in the hole area that can stick to $l t$ due to the fullness. Then there is a valid assembly process $p^{\prime}$ such that the first few steps up to $s t_{i-1}$ are the same and then tile $l t$ instead of $t$ sticks to the supertile $s t_{i-1}$. The process $p^{\prime}$ will finally assemble $s t$ since the TAS $S$ is deterministic. In this case, we have $f\left(p^{\prime}\right) \geq f(p)+1$, which contradicts the property that $f(p)$ is the largest among all assembly of st. Therefore $f(p)=+\infty$.

Now we prove, for the TAS $S$, that for the assembly process wherein all the intermediate supertiles are hole-free, a new tile can stick to the supertile at each step either by two adjacent edges or by an edge of strength at least 2 . If the new tile sticks by more than two edges, then we can pick two adjacent edges. The only remaining case is when the new tile sticks by exactly two parallel edges. We show it is impossible. Without loss of generality, suppose $s t_{i-1}$ becomes $s t_{i}$ by sticking $t$ to $s t_{i-1}$ by North and South sides. There is no tile on the East and on the West sides, or $t$ can stick by two adjacent edges due to the fullness of $s t_{i}$. But in this case, since $s t_{i-1}$ is a derived supertile, there is a tile-path between the two tiles to the North and to the South of $t$ in $s t_{i-1}$. So there is a closed 
tile-path in $s t_{i}$ which encloses either the position to the East or the position to the West of $t$. In other words, $s t_{i}$ contains a hole, which contradicts the fact that $s t_{i}$ is hole-free.

We construct the following right triangular TAS: any square tile $s_{i}$ with glues $\gamma_{1}, \gamma_{2}, \gamma_{3}, \gamma_{4}$ (on East, North, West, South sides, respectively) in $S$ is replaced with four right triangular tiles $\left(i, \gamma_{2}, \gamma_{3}, \mathrm{nw}\right),\left(i, \gamma_{4}, \gamma_{1}, \mathrm{se}\right),\left(i, \gamma_{1}, \gamma_{2}, \mathrm{ne}\right)$ and $\left(i, \gamma_{3}, \gamma_{4}, \mathrm{sw}\right)$, where $i$ is a new glue added with strength $\geq \tau=2$, and when $s_{i}$ is the seed, we let $\left(i, \gamma_{1}, \gamma_{2}\right.$, ne) be the new seed. Then the new right triangular TAS is a division of $S$. Since new tile can stick to the supertile at each step by either two adjacent edges or by an edge of strength at least 2 , the assemble of the square TAS can be simulated by the constructed right triangular TAS. So the constructed right triangular TAS produces a final supertile equivalent to the final supertile of the square TAS.

By Lemmas 1 and 2, we see that square TASs can be simulated by their right triangular divisions only under certain conditions. Now we discuss the other direction: whether every right triangular TAS can be simulated by a square TAS, assuming that the final supertile is square-compatible. For $\tau=1$, the answer is a qualified "yes", as shown by the following lemma.

Lemma 3. For any deterministic right triangular $T A S S_{R}$, at $\tau=1$, if its final supertile is square-compatible and the strength of the glue of the hypothenuse of any of its tiles is 1, then there exists a deterministic square TAS $S$ such that $S_{R}$ is a division of $S$.

Proof. Let $t$ be a tile of $S_{R}$. Without loss of generality, assume that this tile is used at least once in the (unique) final supertile of $S_{R}$. Since the final supertile is square-compatible, there must be a (unique) tile that abuts $t$ in the final supertile, by its hypothenuse. In addition, these two tiles attach to each other via a glue of strength 1 . For each tile $t$ in $S_{R}$, we add to $S$ the square tile obtained by thus binding these two triangular tiles. Being thus contructed, $S$ is deterministic, and $S_{R}$ is its division.

For $\tau=2$, the situation is different, as shown by the following lemma. Note that, in the following lemmas, when comparing two TASs, we ask the final supertiles generated by them to be equivalent, that is, to have the same shape and the same border glues.

Lemma 4. There exists a deterministic right triangular TAS at $\tau=2$, whose final supertile is square-compatible, but no deterministic square TAS produces an equivalent final supertile.

An example of a right triangular TAS at $\tau=2$ as postulated in Lemma 4 is illustrated in Fig. 13 (right), where each tile is numbered in the order of a possible assembly process. Note that any square TAS that produces a supertile of the same shape as the rightmost supertile depicted in Fig. 13 must include a square tile with West side glue a, and South glue b. However, if a tile system 


\begin{tabular}{|c||c||c|}
\hline 1 & 3 & 5 \\
\hline \hline$S$ & & 7 \\
\hline \hline 2 & 4 & 6 \\
\hline
\end{tabular}

\begin{tabular}{|c||c||c||c|}
\hline 1 & 3 & 5 & 7 \\
\hline \hline$S$ & 15 & 13 & 9 \\
\hline \hline 2 & 14 & 12 & 11 \\
\hline \hline 4 & 6 & 8 & 10 \\
\hline
\end{tabular}

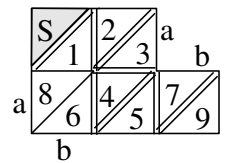

Fig. 13. Examples that show that square TASs and triangular TASs are, in some sense, not comparable from the point of view of the shapes of final supertiles they generate. The left and center figures depict two final supertiles of square systems at $\tau=2$, and $\tau=3$, respectively, that illustrate Lemma 10 The right figure $(\tau=2)$, illustrates Lemma 4 Each glue, unless mentioned, is unique, and thus, the label is omitted.

contained such a tile, its assembly would grow at its North-East corner and produce the $3 \times 2$ rectangle instead.

Lemmas 1 and 4 indicate that square TASs and triangular TASs are, in some sense, not comparable from the point of view of the shapes of final supertiles they generate.

The following lemma compares square TASs with hexagonal TASs from the point of view of the shapes of the final supertiles they generate.

Lemma 5. No supertile is both hexagon-compatible and square-compatible, even under possible affine transformation on $\mathcal{R}^{2}$.

Proof. Suppose there is a supertile st assembled by hexagonal tiles that is of the same shape to a supertile $s t^{\prime}$ assembled by square tiles under affine transformation $F$. Let $t$ be the left-most tile on the top-most row of tiles in st. Assume the vertices of $t$ are $v_{1}, \ldots, v_{6}$, starting from the top-right vertex in the counterclockwise order. Then $v_{1}, v_{2}, v_{3}, v_{6}$ are also vertices of the supertile $s t$ by the position of $t$. Assume $v_{1}^{\prime}, v_{2}^{\prime}, v_{3}^{\prime}, v_{6}^{\prime}$ be the corresponding vertices in $s t^{\prime}$. Since an affine transformation transform lines to lines, the angle $v_{1}^{\prime}, v_{2}^{\prime}, v_{3}^{\prime}$ and $v_{2}^{\prime}, v_{1}^{\prime}, v_{6}^{\prime}$ are of degree $\pi / 4$ or $3 \pi / 4$. In other words, the two lines $v_{2}^{\prime} v_{3}^{\prime}$ and $v_{1}^{\prime} v_{6}^{\prime}$ are parallel. Then $v_{2} v_{3}$ and $v_{1} v_{6}$ should also be parallel, since an affine transformation preserves parallel relationship of lines. But $v_{2} v_{3}$ and $v_{1} v_{6}$ are not parallel, a contradiction. So no hexagon-compatible shape is square-compatible even under possible affine transformation on $\mathcal{R}^{2}$.

We now compare triangular TASs with hexagonal TASs from the point of view of the shapes of the final supertiles they generate.

An equilateral triangular TAS $T$ is called a triangular division of a hexagonal TAS $H$, if ( $i$ ) for any hexagonal tile $h$ in $H$, there are six triangular tiles in $T$ that can attach to each other to produce a supertile equivalent to $h$ at temperature $\tau \geq 1$, and (ii) for any triangular tile $t$ in $T$, there are five other triangular tiles in $T$, that can attach to each other to produce a supertile equivalent to a hexagonal tile in $H$ (see Fig. 10). An equilateral triangular TAS $T$ is called 
a deterministic triangular division of a hexagonal TAS $H$ if it is a triangular division of $H$ that is a deterministic TAS. A right triangular TAS $T$ is called a division of a hexagonal TAS $H$, if $T$ is the flattening $\mathcal{F}\left(T^{\prime}\right)$ of an equilateral triangle TAS $T^{\prime}$ that is a division of $H$. A right triangular TAS $T$ is called a deterministic division of a hexagonal TAS $H$ if it is a division of $H$ that is a deterministic TAS.

It is obvious that for any hexagonal TAS $H$ at $\tau=1$, there is a triangular division of $H$ that produces a final supertile of the same shape. For $\tau=2$ the situation is different, as shown by the following lemma.

Lemma 6. There exists a deterministic hexagonal TAS $H$ at $\tau=2$, such that no deterministic triangular division of $H$ produces an equivalent final supertile.

An example is illustrated in Fig. 14 (left), for $\tau=2$, where $S$ is the seed and tile 1 attached to the supertile before tile 2 is. Tiles $s$ and 1 can be simulated by their triangular divisions, but tile 2 cannot, since the cooperation between edges, even for the case when the cooperative edges abut in the hexagonal tile, cannot be preserved when replacing a hexagonal tile by equilateral triangular tiles. Indeed, not triangular tile from the division of the hexagonal tile can attach to the supertile formed by the seed and tile 1 , since the edges that would be needed for any of them to attach all have strength 1 . This simple example shows that most hexagonal TASs cannot be simulated by their triangular divisions.

It is obvious that for any equilateral triangular TAS $T$ at $\tau=1$ whose final supertile is hexagon-compatible, there is a hexagonal TAS $H$ that produces an equivalent final supertile. For $\tau=2$, the situation is different, as shown by the following lemma.

Lemma 7. There exists a deterministic equilateral triangular TAS $T$ at $\tau=2$ whose final supertile is hexagon-compatible, but no deterministic hexagonal TAS produces an equivalent final supertile.

An example is illustrated in Fig.14(right), where tiles are numbered in a possible order of assembly. Note that any hexagonal TAS that produces an equivalent final supertile as that of Fig. 14 (right), has to contain a hexagonal tile $t$ such that $\gamma_{1}(t)=b$ and $\gamma_{2}(t)=a$. Then the process of assembly of such a hexagonal TAS can grow further in the right-bottom direction, and thus cannot produce the unique required final supertile.

By replacing pairs of tiles $(s, 1),(2,3), \ldots,(10,11)$ by diamond tiles in the example in Fig. 14, one can also show that there exists a diamond TAS whose final supertile is hexagon-compatible but no hexagonal TAS produces an equivalent final supertile.

Lemmas 6 and 7 together indicate that hexagonal TASs and triangular TASs are, in some sense, not comparable from the point of view of the shapes of the final supertiles they generate.

Let us now compare square with diamond tile assembly systems. For every square TAS $S$, there is a diamond TAS $D$ such that the final supertiles produced by the systems are equivalent up to an affine transformation on the two 

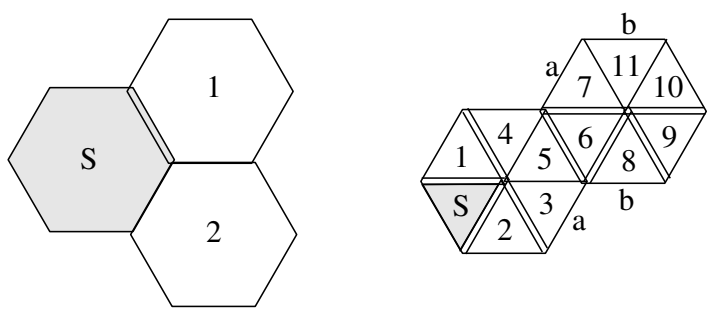

Fig. 14. Examples that show that hexagonal TASs and triangular TASs are, in some sense, not comparable from the point of view of the final supertiles they can generate. The left figure is a hexagonal system at $\tau=2$ that illustrates Lemma 6 The right figure $(\tau=2)$ illustrates Lemma 7 Each glue, unless mentioned, is unique, and thus, the label is omitted.

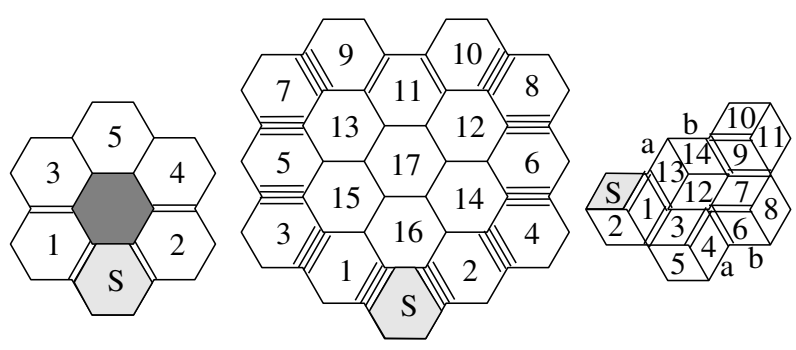

Fig. 15. Examples that show that hexagonal TASs and diamond TASs are, in some sense, not comparable from the point of view of the final supertiles they can generate. The left and center figures depict two hexagonal systems, at $\tau=2$ and $\tau=4$ respectively, that illustrate Lemma 8 The right figure $(\tau=2)$ illustrates Lemma 9 . Each glue, unless mentioned, is unique, and thus, the label is omitted.

dimensional plane $\mathcal{R}^{2}$. To see this, we use a single type, either II, IV, or VI, of diamond tile to simulate each square tile. On the other hand, by Lemma 5 , every diamond TAS that produces a hexagon-compatible final supertile cannot be simulated by a square TAS.

The comparison of diamond TAS and equilateral TAS is similar to the comparison of square TAS and right triangular TAS: the examples given in Fig. 13, under affine transformations, indicate that diamond TASs and triangular TASs are, in some sense, not comparable from the point of view of the shapes of the final supertiles they generate.

Now we compare diamond TASs with hexagonal TASs. A diamond TAS $D$ is called a diamond division of a hexagonal TAS $H$ if (i) for any hexagonal tile $h$ in $H$, there are three diamond tiles in $D$ that can can assemble to produce a supertile equivalent to $h$ at temperature $\tau \geq 1$, and (ii) for any diamond tile $d$ in $D$, there are other two diamond tiles in $D$ that can assemble to produce a supertile tile equivalent to a hexagonal in $H$ (see Fig. 11). A diamond TAS $D$ is 
called a deterministic diamond division of a hexagonal TAS $H$ if it is a diamond division of $H$ that is a deterministic TAS.

Lemma 8. There exists a deterministic hexagonal TAS $H$ such that no determinisic diamond division of $H$ produces an equivalent final supertile.

Two examples that prove the statement of Lemma 8, are illustrated in Fig. 15, one for $\tau=2$ (left), and one for $\tau=4$ (centre), none of which can be simulated by their diamond division. Each tile is numbered in the order in which it would appear in a possible assembly process. The two examples are of the same flavor as those in Fig. 13 tile 5, respectively tile 11, cannot be simulated by successive attachments of diamond tiles, since the two cooperative sticky edges that attached the original hexagonal tile to the supertile are not adjacent, hence they will belong to different diamond tiles in the division.

Lemma 9. There exists a deterministic diamond TAS T whose final supertile is hexagon-compatible, but no deterministic hexagonal TAS produces an equivalent final supertile.

An example is given in Fig. 15 (right). The proof is similar to that of Lemma 7 .

Lemmas 8 and 9 indicate that hexagonal TASs and diamond TASs are, in some sense, not comparable from the point of view of the shapes of the final supertiles they generate. In spite of this, under certain conditions, hexagonal TASs can be simulated by diamond TASs. It is, for example, obvious that for any hexagonal TAS $H$ at $\tau=1$, there is a diamond division of $H$ that produces a final supertile of the same shape. Furthermore, we have the following result.

Proposition 3. For any hexagonal TAS $H$ at $\tau=1$, and any hexagonal TAS at $\tau=2$, whose final supertile has no hole, there is a diamond division of $H$ that produces a final supertile of the same shape.

The proof is similar to that of Lemma 2 ,

\section{Conclusion}

Square tile assembly systems have been widely studied in the literature as a model, in particular for DNA self-assembly. In this paper, we focus instead on triangular and hexagonal TASs and some of their properties. We show that, in some restricted sense, triangular TASs and square TASs, respectively triangular TASs and hexagonal TASs, are not comparable from the point of view of the shape of the final supertiles they generate. More precisely, there exists a deterministic square (respectively hexagonal) TAS $S$ such that no deterministic triangular division of $S$ produces an equivalent final supertile (of the same shape and same boundary glues). Also, there exists a deterministic triangular TAS T such that the final supertile is square (respectively hexagon)-compatible, but no deterministic square (respectively hexagonal) TAS produces an equivalent final supertile. 
Acknowledgements. We thank Dr. David Doty for his valuable comments on the earlier versions of the paper. This research was supported by The Natural Sciences and Engineering Council of Canada Discovery Grant R2824A01 and Canada Research Chair Award to Lila Kari.

\section{References}

1. Adleman, L.: Toward a mathematical theory of self-assembly (manuscript, 2000), https://eprints.kfupm.edu.sa/72519/1/72519.pdf

2. Winfree, E., Liu, F., Wenzler, L.A., Seeman, N.C.: Design and self-assembly of two-dimensional DNA crystals. Nature 394, 539-544 (1998)

3. Wang, H.: Proving theorems by pattern recognition II. Bell System Technical Journal 40, 1-42 (1961)

4. Winfree, E.: On the computational power of DNA annealing and ligation. In: DNA Based Computers: DIMACS Workshop, vol. 27, pp. 199-221 (1996)

5. Rothemund, P.W.K., Winfree, E.: The program-size complexity of self-assembled squares. In: Proc. 32nd Ann. ACM Symp. Theor. of Comp. (STOC 2000), pp. 459-468 (2000)

6. Adleman, L., Cheng, Q., Goel, A., Huang, M.: Running time and program size for self-assembled. In: Proc. 33rd Ann. ACM Symp. Theor. of Comp. (STOC 2001), pp. $740-748$ (2001)

7. Kao, M., Schweller, R.: Reducing tile complexity for self-assembly through temperature programming. In: Proc. 7th Ann. ACM-SIAM Symp. Discrete Algorithms (SODA), pp. 571-580 (2006)

8. Liu, D., Wang, M., Deng, Z., Walulu, R., Mao, C.: Tensegrity: Construction of rigid DNA triangles with flexible four-arm DNA junctions. J. Am. Chem. Soc. 126, 2324-2325 (2004)

9. Ding, B., Sha, R., Seeman, N.C.: Pseudohexagonal 2D DNA crystals from double crossover cohesion. J. Am. Chem. Soc. 126, 10230-10231 (2004)

10. He, Y., Chen, Y., Liu, H., Ribbe, A.E., Mao, C.: Self-assembly of hexagonal DNA two-dimensional (2D) arrays. J. Am. Chem. Soc. 127, 12202-12203 (2005)

11. Rothemund, P.W.K., Papadakis, N., Winfree, E.: Algorithmic self-assembly of DNA Sierpinski triangles. PLoS Biol. 2, e424 (2004), http://dx.doi.org/10.1371/journal.pbio.0020424 